\title{
Evaluation De L'impact De La Pollution Agricole Sur La Qualite Des Eaux Souterraines De La Nappe Du Gharb
}

\author{
Bouderka Nouzha, Dr. \\ Souid Ahmed Kacem, Prof. \\ Lakhili Ferdaouss, Dr. \\ Lahrach Abrerrahim, Prof. \\ Benabdelhadi Mohammed, Prof. \\ Laboratoire des Géo-Ressources et Environnement \\ Université Sidi Mohammed Ben Abdellah, Fès, Maroc
}

doi: 10.19044/esj.2016.v12n11p509 URL:http://dx.doi.org/10.19044/esj.2016.v12n11p509

\begin{abstract}
The Gharb plain is located in the North West of Morocco and corresponds to a vast plain of the Sebou watershed. It includes an irrigable perimeter of a 250,000 ha, of which more than 120,000 ha are designed as big hydraulics to meet the drinking water and irrigation needs of the region. The intensification of agriculture in this region leads to a significant use of phytosanitary products, which can impact the physico-chemical quality of the Gharb groundwater. To assess this quality we proceeded to the analysis of the physico-chemical parameters $(\mathrm{pH}$, electric conductivity, dissolved oxygen, chlorides, sodium, potassium, nitrates, ammonium, and orthophosphates) and the study of the metallic contamination of waters of 17 wells located in the region of Sidi Kacem-Sidi Slimane. The results of performed analysis, revealed a pollution of an agricultural origin. This is confirmed by the maximum levels of chlorides, which exceed 2,734 mg/l, thereby explaining the high conductivity levels $(5000 \mu / \mathrm{cm})$. Nevertheless, a lithological influence cannot be excluded. The nitrate levels $(160 \mathrm{mg} / \mathrm{l})$ indicate a nitric pollution of underground waters. The heavy metals measured showed a metallic contamination exceeding the norms of lead and aluminium concentration, elements which may have their origin in fertilizers undergoing an excessive use in the area. These results confirm the impact of the agricultural intensification in the degradation of the quality of the underground water of the Gharb region.
\end{abstract}


Keywords: Gharb, agricultural pollution, groundwater, physico-chemical parameters, nitrates, metals

\section{Resumé}

La plaine du Gharb est située dans la partie nord-ouest du Maroc et correspond à une vaste cuvette du bassin versant du Sebou. Elle comprend un périmètre irrigable d'une superficie de 250000 ha, dont plus de 120000 ha sont actuellement aménagés en grande hydraulique pour répondre au besoin de la région en eau potable et d'irrigation. L'intensification de l'agriculture dans cette région entraine une utilisation importante de produits phytosanitaires. Plusieurs études ont signalé un impact sur la qualité physicochimique des eaux de la nappe phréatique du Gharb. Pour évaluer l'état actuel de la qualité nous avons procédé à l'analyse des paramètres physicochimiques ( $\mathrm{pH}$, conductivité électrique, oxygène dissout, chlorures, sodium, potassium, nitrates, ammonium, orthophosphates) et à l'étude de la contamination métallique des eaux de 17 puits situés dans la région de Sidi Kacem-Sidi Slimane. Les résultats des analyses effectuées, ont révélé une pollution chlorée des eaux de la nappe. Ceci est confirmé par des teneurs élevées en chlorures, qui dépassent les $2734 \mathrm{mg} / \mathrm{l}$, ce qui explique les valeurs élevées de la conductivité $(5000 \mu / \mathrm{cm})$. Si une pollution d'origine agricole est évoquée, on ne peut négliger la contribution lithologique sur les résultats obtenus. Les teneurs en nitrates (160 mg/l), indiquent une pollution évidente des eaux souterraines. Les métaux lourds dosés ont montré une contamination métallique qui dépasse les normes de la concentration en plomb et en aluminium, dont l'origine se trouve, entre autres, dans les engrais et les fertilisants. Ces résultats confirment l'impact de l'intensification de l'agriculture dans la dégradation de la qualité des eaux souterraines de la région du Gharb.

Mots clés: Gharb, pollution agricole, eaux souterraines, paramètres physicochimiques, nitrates, métaux

\section{Introduction}

La plaine du Gharb, constitue l'un des principaux périmètres irrigués du Maroc avec une superficie totale irrigable de 250000 ha, soit $16 \%$ du potentiel national de l'irrigation. L'intensification des cultures, par la mauvaise gestion de l'irrigation et l'utilisation accrue d'engrais et de pesticides, s'est traduite par une altération sensible de la qualité des sols et des milieux récepteurs (eaux de surface et eaux souterraines). Les études physicochimiques des eaux souterraines menées dans cette plaine au cours des dernières décennies, mettent en évidence une pollution par les nitrates 
qui s'accentue sensiblement avec le temps (Kholtei, 2002; Kholtei et al., 2003; Moullard et Hazan , 1960 ; Daifi et al., 2014).

La présente étude concerne l'évaluation de la qualité physicochimique des eaux souterraines de la partie orientale de la nappe du Gharb (région Sidi Kacem et Sidi Slimane). Pour ce faire, une campagne d'échantillonnage a été entreprise en novembre 2014. Les paramètres physico-chimiques, mesurés in situ et au laboratoire, ont servi pour la caractérisation de ces eaux.

\section{Présentation de la zone d'étude}

L'aire d'étude fait partie de la plaine du Gharb qui est située au nordouest du Maroc et couvre une superficie de l'ordre de 616000 ha. Elle est entourée par l'Océan Atlantique, les dunes du Sahel, les collines du pré- Rif et le plateau Maamora avec une altitude qui varie entre 4 et $25 \mathrm{~m}$ (fig. 1). Elle est composée d'une bande côtière (cordon dunaire, dépressions inondées, dunes intérieures), de bordures continentales et d'une plaine alluviale qui appartient à la plaine centrale du Sebou qui constitue le principal oued. Elle renferme un système aquifère complexe constitué de deux nappes superposées qui sont la nappe phréatique au centre de la plaine et la nappe profonde qui affleure sur les pourtours sud et ouest. Le substratum général de ce complexe aquifère est constitué par le toit de l'épaisse série marneuse mio-pliocène dite communément «marnes bleues» (Saaidi, 1988).

La plaine du Gharb est formée d’une épaisse série sédimentaire de dépôts très hétérogènes depuis les marnes du Miocène jusqu'aux limons du Quaternaire récent. Son climat passe de sub-humide à hiver tempéré en zone côtière à semi-aride à hiver chaud à l'intérieur de la plaine. La zone est à vocation agricole et a fait l'objet d'aménagements hydrauliques considérables et de mise en valeur agricole depuis les années 30 (Daniane et al., 1994). 


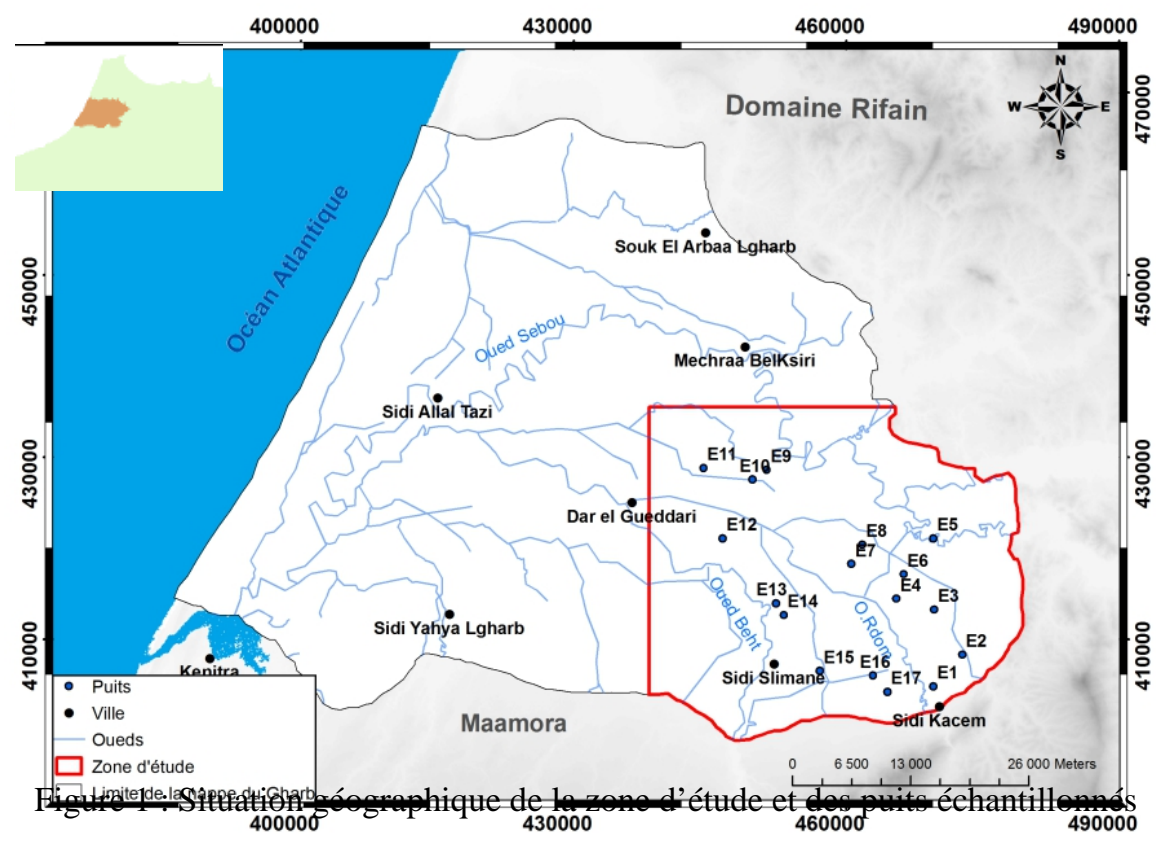

\section{Matériels et Méthodes}

Les points d'eau échantillonnés ont été choisis de telle manière à couvrir l'ensemble de la partie sud-est de la nappe du Gharb. Au total dixsept prélèvements pour les analyses physico-chimiques ont été effectués en novembre 2014. Les échantillons ont été prélevés dans des contenants de 1 litre en plastique, préalablement rincées avec l'eau de chaque puits échantillonné. Ils ont été ensuite conservés à $4^{\circ} \mathrm{C}$ pendant le transport, puis ont été analysés dans les 24 heures qui suivent. Ces points d'eau ont été localisés par leurs coordonnées Lambert déterminées par GPS. Les paramètres physico-chimiques $(\mathrm{pH}$, conductivité électrique, salinité et oxygène dissout) ont été mesurés in situ à l'aide d'une sonde multiparamètres.

Les chlorures sont dosés par la méthode volumétrique de Mohr en présence du nitrate d'argent. Les nitrates sont déterminés par la méthode spectrophotométrique en présence de salicylate de sodium. Ces analyses ont été analysées au laboratoire Géo-Ressources et Environnement de la Faculté des Sciences et Technique de Fès. Les éléments en traces, sodium et potassium, ont été effectués à l'aide de la spectrométrie d'émission couplée à un plasma induit (ICP-AES) au laboratoire du CURI de l'université Sidi Mohammed Benabdellah de Fès. Les résultats relatifs à ces échantillons sont présentés sous forme de cartes et de graphes. 


\section{Résultats et discussions \\ Potentiel Hydrogène pH}

Le $\mathrm{pH}$ de l'eau mesure la concentration des protons $\mathrm{H}^{+}$contenus dans l'eau. Il résume la stabilité de l'équilibre établi entre les différentes formes de l'acide carbonique et il est lié au système tampon développé par les carbonates et les bicarbonates (Ezzaouaq, 1991; El Blidi et Fekhaoui 2003; Himmi et al., 2003).

Les valeurs du $\mathrm{pH}$ des eaux échantillonnées varient entre 6,6 et 7,65 ce qui les qualifie comme proches de la neutralité. En se référant aux normes marocaines $(6.5<\mathrm{pH}<8.5)$ pour l'eau potable, on remarque que $100 \%$ des eaux analysées sont conformes à la consommation (fig.2).

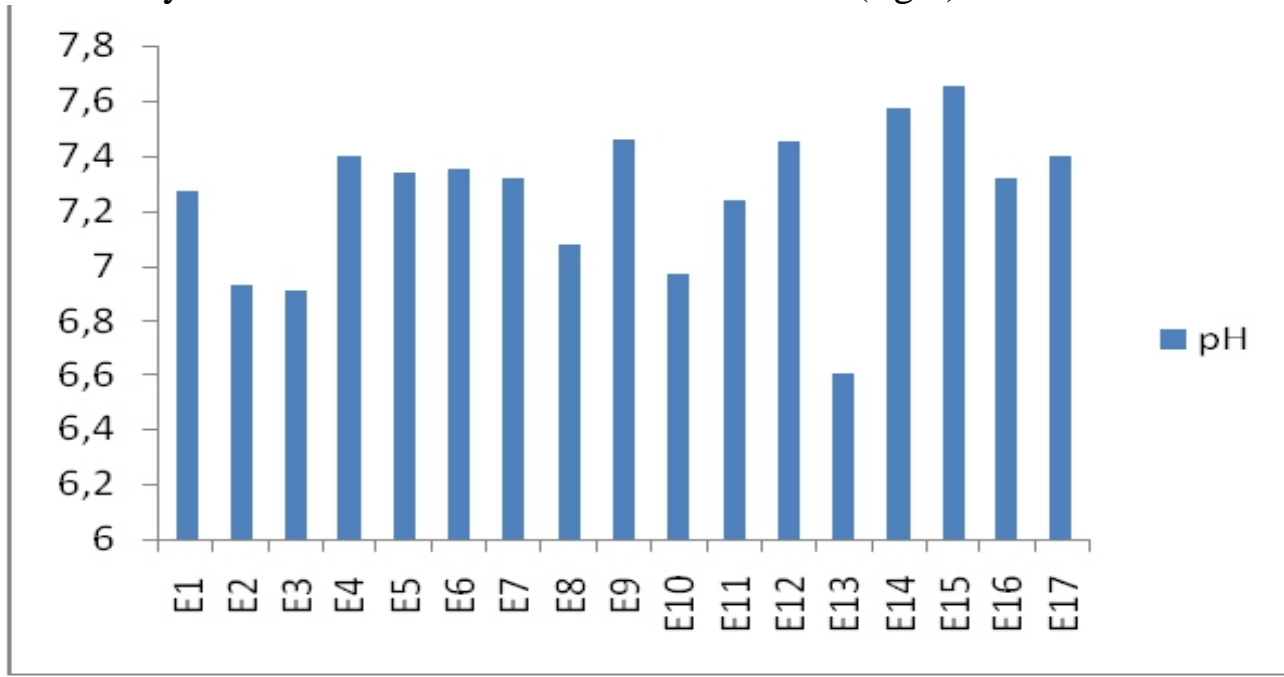

Figure 2 : Variation spatiale du $\mathrm{pH}$ de l'eau

\section{Conductivité Electrique}

La conductivité électrique désigne la capacité de l'eau à conduire un courant électrique. Elle est déterminée par la teneur en substances dissoutes, la charge ionique, la capacité d'ionisation, la mobilité et la température de l'eau. Ainsi, la mesure de la conductivité permet d'apprécier la quantité des sels dissous dans l'eau et par conséquent sa minéralisation (Haddad-et Ghoualem, 2014).

Les eaux des points étudiés sont minéralisées (fig.3), avec des valeurs qui oscillent entre 770 et $9890 \mu \mathrm{s} / \mathrm{cm}$. 53\% des échantillons dépassent la valeur maximale admissible (VMA) qui est fixée à $2700 \mu \mathrm{s} / \mathrm{cm}$ selon les normes marocaines de la potabilité (E1, E5, E7, E8, E9, E10, E12, E14 et E17). 


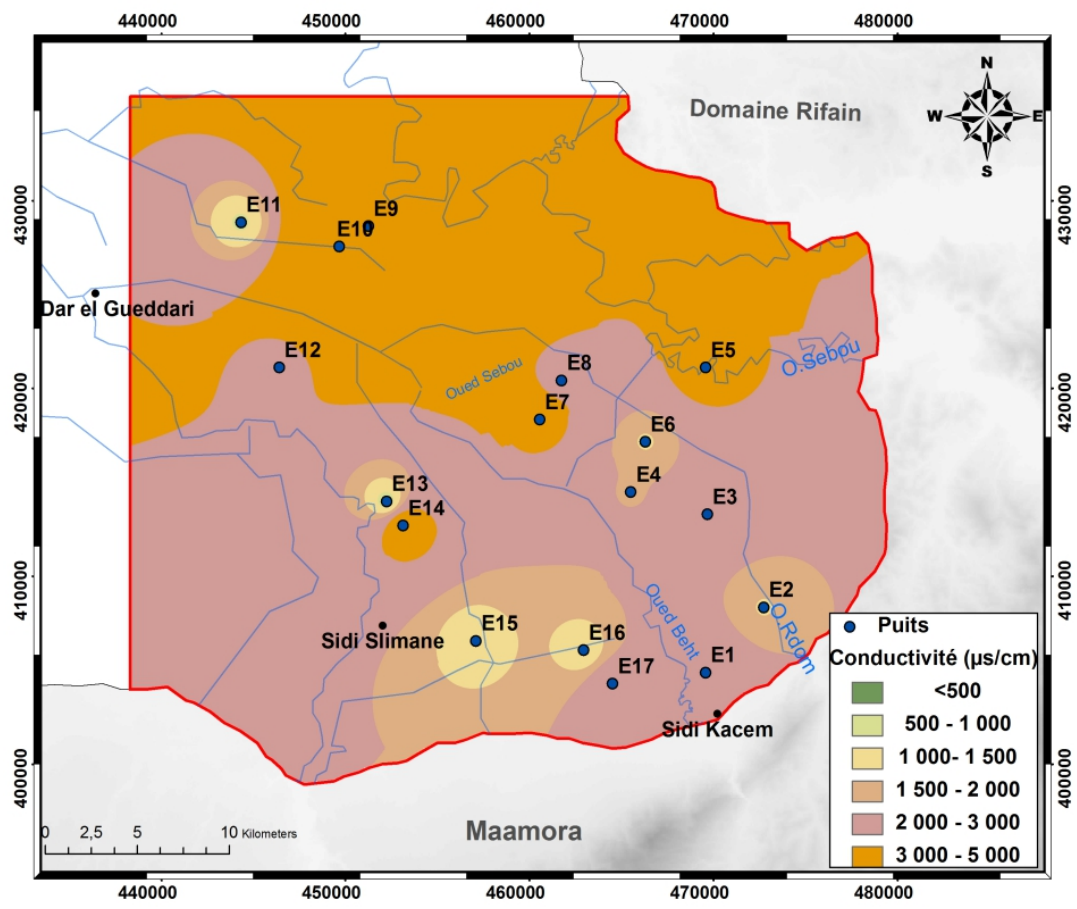

Figure 3 : Répartition spatiale de la conductivité électrique.

\section{Oxygène dissout}

L'oxygène dissout est un paramètre qui renseigne sur l'état de l'eau du puits et d'autre part il favorise la croissance des micro-organismes qui dégradent la matière organique. En général, les valeurs faibles favorisent le développement des germes pathogènes (HCEFLCD, 2007).

Pour l'ensemble des prélèvements, l'oxygène dissout varie de 0,4 à $6,2 \mathrm{mg} / \mathrm{l}$ durant la période d'étude et les résultats obtenus montrent que les puits sont légèrement sous oxygénés.

\section{Chlorures}

Les chlorures sont largement répandus dans la nature, généralement sous forme de sels de sodium $(\mathrm{NaCl})$ et de potassium $(\mathrm{KCl})$. Ils sont souvent utilisés comme indice de pollution (N’Diaye et al. 2014). Les chlorures existent dans toutes les eaux à des concentrations très variables.

Les teneurs en chlorures des échantillons d'eau analysés (fig.4) affichent des valeurs oscillant entre $110 \mathrm{mg} / \mathrm{l}$ et $2734 \mathrm{mg} / \mathrm{l}$. Les points d'eau situés en amont de la plaine du Gharb sont conformes aux normes marocaines des eaux souterraines, étant donné que la concentration en chlorures est inférieure à $750 \mathrm{mg} / \mathrm{l}$. Vers l'aval de la plaine, les points E9 et E10 montrent des teneurs qui dépassent les normes, soit 2734 et $1422 \mathrm{mg} / \mathrm{l}$. Cette composition minérale élevée peut être expliquée par l'utilisation des 
engrais mais aussi par une présence naturelle due aux dépôts du Permo-Trias à caractère évaporitique (salifère et gypsifère). Un autre facteur naturel qui pourrait aussi expliquer ces teneurs, c'est l'évaporation des nappes perchées qui caractérise les horizons supérieurs des aquifères du Gharb. Ces résultats sont en adéquation avec les études hydrochimiques menées par la Direction Générale de l’Hydraulique (1994) qui ont montré que les eaux souterraines de la plaine du Gharb sont caractérisées par un faciès chloruré sodique.

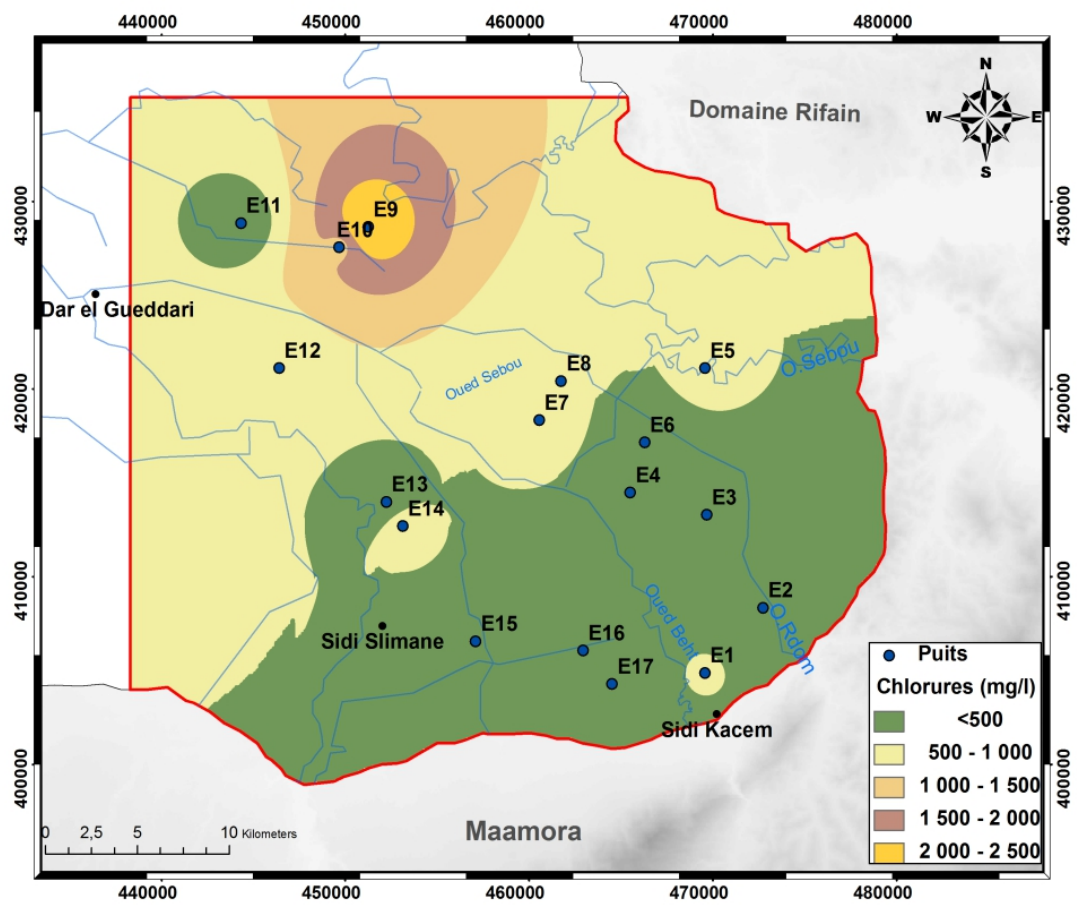

Figure 4: Répartition spatiale des chlorures

\section{Sodium}

L'analyse des données a montré que les teneurs moyennes en sodium dans les eaux des points étudiés varient de $60 \mathrm{mg} / \mathrm{l}$ à $655 \mathrm{mg} / \mathrm{l}$. Nous avons remarqué que les points situés vers l'aval dépassaient les normes de potabilité. L'augmentation du sodium dans l'eau souterraine à des concentrations supérieures à la norme peut indiquer la présence d'agents polluants ou une invasion d'eau salée.

Les teneurs élevées en sodium des eaux de la nappe ont la même origine que celle des chlorures. En effet, le sodium accompagne généralement la pollution due aux chlorures (Pesson, 1976). Il y a toujours une corrélation linéaire entre ces deux éléments (fig. 5) 


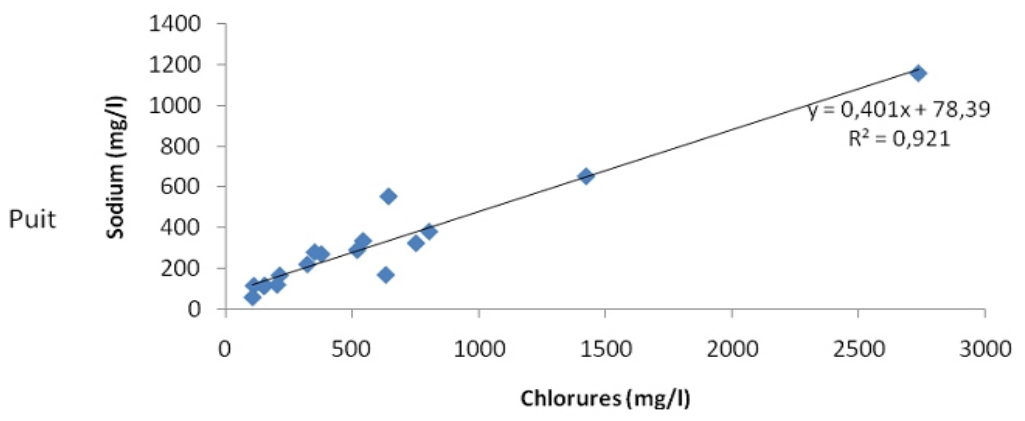

Figure 5: Courbe de corrélation entre les concentrations des chlorures et du sodium des eaux souterraines.

\section{Potassium}

Le potassium est généralement l'élément majeur le moins abondant dans les eaux après le sodium, le calcium et le magnésium ; il ne prend qu'exceptionnellement le troisième rang des cations. Le potassium est un élément indispensable à la vie et notamment à la croissance des végétaux. En agriculture, il est utilisé comme engrais sous forme de sulfate de potassium, de chlorure de potassium, ou encore de nitrate de potassium (Pesson-1976).

La teneur en potassium est presque constante dans les eaux naturelles, elle ne dépasse pas habituellement 10 à $15 \mathrm{mg} / \mathrm{l}$ (Haddad-et Ghoualem, 2014). Sa concentration dans les points d'eau contrôlés (fig.6) varie entre $1,77 \mathrm{mg} / \mathrm{l}$ et $16,50 \mathrm{mg} / \mathrm{l}$; elle ne dépasse pas les normes marocaines de potabilité fixées à $20 \mathrm{mg} / \mathrm{l}$. La valeur la plus élevée en potassium est enregistrée au niveau du point d'eau E6.

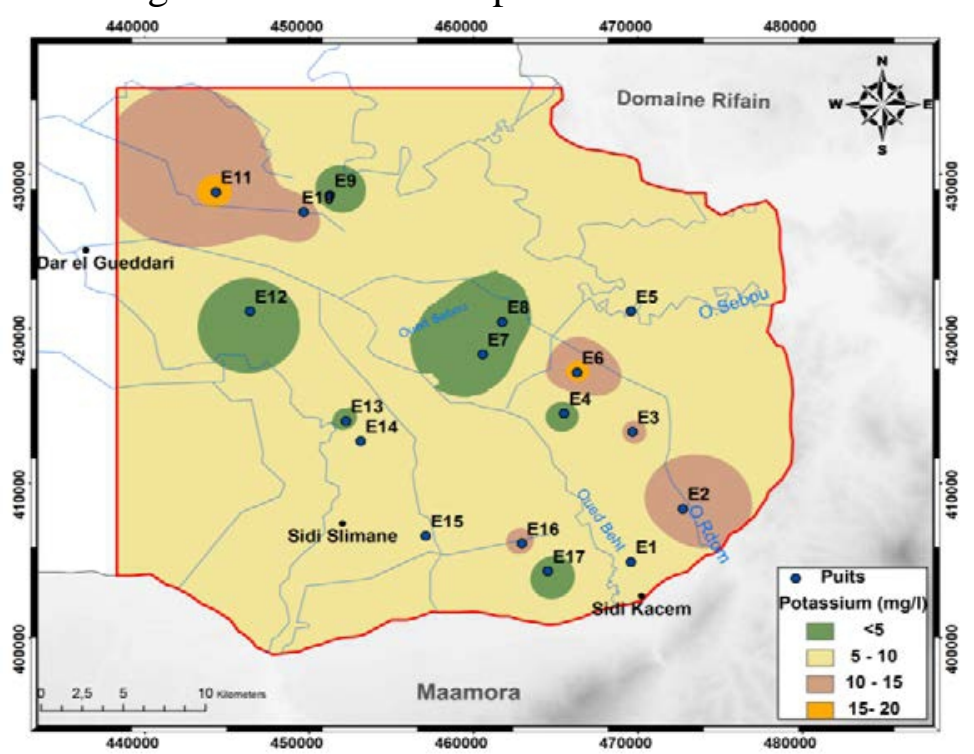

Figure 6 : Répartition spatiale du potassium 


\section{Nitrates}

Les nitrates sont importants à suivre. En effet, retrouvés en excès, ils sont un marqueur incontestable de pollution. Au niveau des eaux étudiées, la concentration moyenne en nitrates est de l'ordre de 27,89 mg/l ce qui est conforme à la norme de $50 \mathrm{mg} / \mathrm{l}$ fixée par l’OMS (1986), en terme de potabilité.

Les teneurs en nitrates varient entre 0,031 et $160 \mathrm{mg} / \mathrm{l}$, elles sont en général conformes aux normes, sauf pour les puits E9 et E1 (fig.7). Des valeurs élevées ont été enregistrées à Mnasra (Bouchouata et al, 2011) et dans la ville de Kénitra (Daifi et al. 2014). Cette altération de la qualité de ces deux puits par les nitrates pourrait être attribuée aux rejets ponctuels et dispersés des produits d'élevage et par l'usage des fertilisants chimiques et des pesticides lié aux activités agricoles, qui s’infiltrent par la suite à travers le sol jusqu'à la nappe phréatique. Bien que les nitrates n’aient pas d'effets toxiques directs sauf à des doses élevées, ils se transforment facilement par réduction bactérienne en nitrites, dont les effets sur la santé ne seraient pas neutres (Elbouqadaoui et al. 2009).

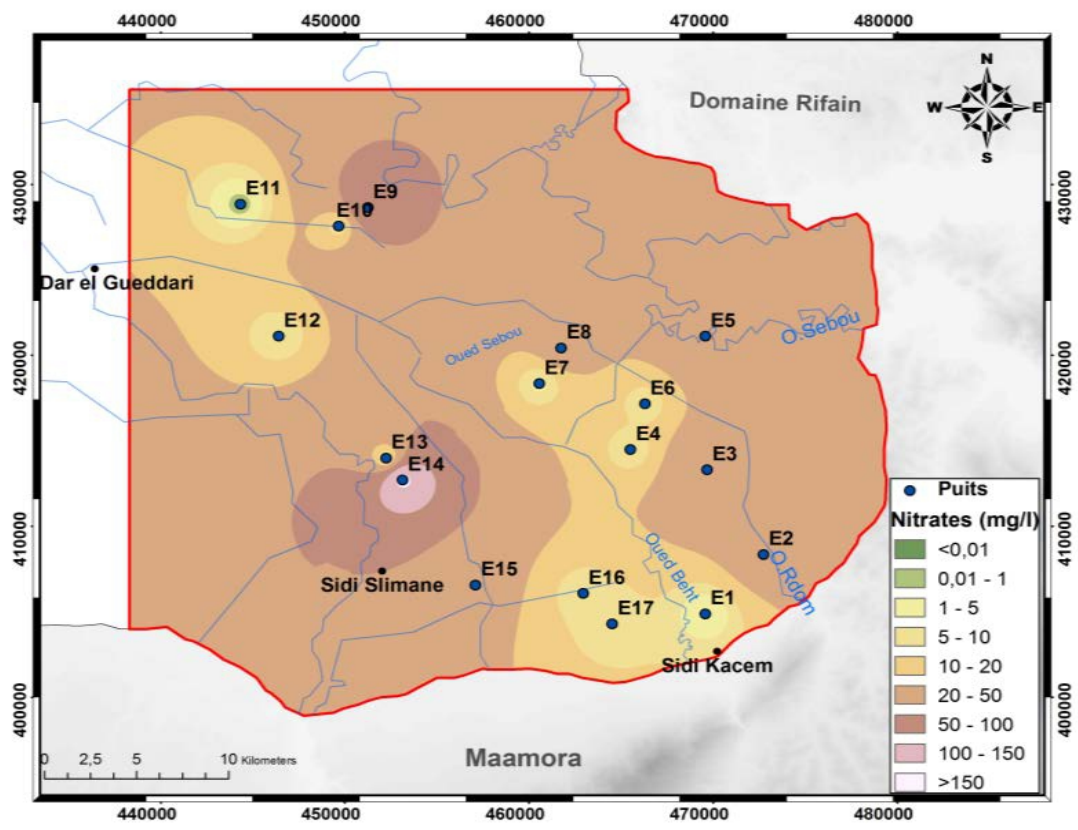

Figure 7: Répartition spatiale des nitrates

\section{Ammonium $\mathrm{NH}_{4}^{+}$}

L’azote ammoniacal constitue un des maillons du cycle complexe de l'azote dans son état primitif. C'est un gaz soluble dans l'eau. Il constitue un bon indicateur de pollution des cours d'eau par des rejets organiques d'origine agricole, domestique ou industrielle (Ben Abbou et al, 2014). 
Dans notre zone d'étude, les teneurs en Ammonium sont globalement satisfaisantes et varient entre un minimum de $0,017 \mathrm{mg} / \mathrm{l}$ au niveau du puits E1 et un maximum de $1,059 \mathrm{mg} / \mathrm{l}$ au niveau du puits E17. La valeur maximale admissible est fixée à $0,5 \mathrm{mg} / \mathrm{l}$

\section{Orthophosphates}

Les orthophosphates constituent la forme prédominante du phosphore disponible dans le sol ou dans l'eau. En combinaison avec l'azote, le phosphore est utilisé dans les engrais chimiques et naturels pour enrichir les sols agricoles. Au cours des dernières années, une attention particulière a été apportée à cet élément afin de mieux contrôler la fertilisation agricole (Polan et Monique, 1990).

Les teneurs des orthophosphates dans les puits étudiés varient entre une valeur minimale de $0.002 \mathrm{mg} / \mathrm{l}$ et une maximale de $0,216 \mathrm{mg} / \mathrm{l}$. Ces valeurs restent très faibles et inférieures à la valeur maximale admissible fixée à $0,5 \mathrm{mg} / \mathrm{l}$.

\section{Métaux lourds}

Le dosage des métaux lourds ( $\mathrm{Pb}, \mathrm{Al}, \mathrm{As}, \mathrm{Cd}, \mathrm{Cr}, \mathrm{Cu}, \mathrm{Fe}, \mathrm{Ni}, \mathrm{Zn}$ ) montrent que les eaux des puits étudiés contiennent en général des teneurs très négligeables, à l'exception des puits E2, E16 et E17 qui présentent des taux qui dépassent la valeur maximale admissible (VMA) des eaux souterraines selon la norme marocaine. E2 présente une pollution en plomb $(0,133 \mathrm{mg} / \mathrm{l})$ alors que E16 et E17 présentent une pollution due au taux élevé en aluminium (0,334 et 0,452 mg/l) (fig.8). La présence de ces métaux même à de faibles concentrations dans les eaux peut avoir des conséquences néfastes sur le plan écologique et sanitaire (OMS, 2013).

Cette contamination peut être due à l'utilisation excessive et à fortes doses des engrais et aux rejets des produits d'élevage.

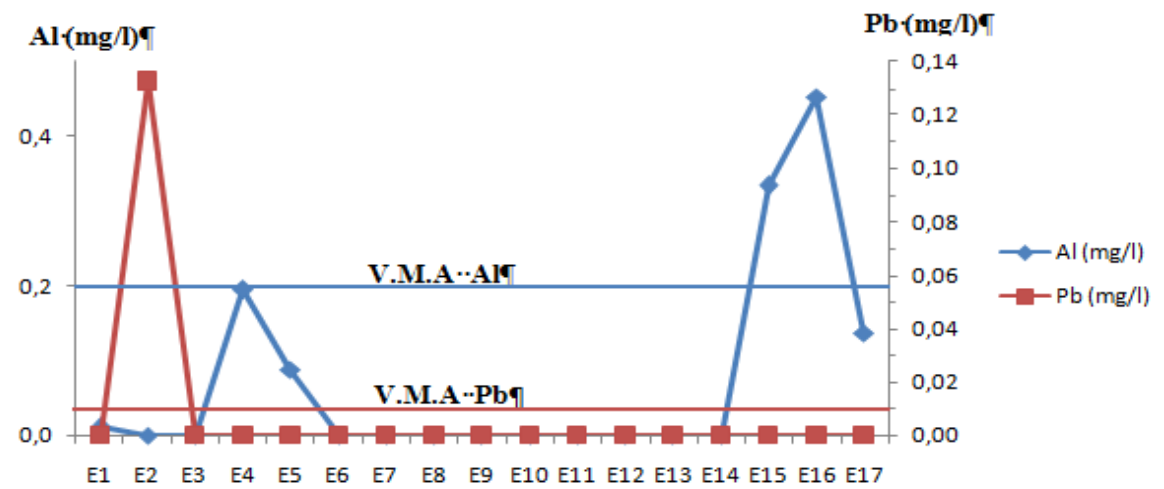

Figure.8: Evolution spatiale de la concentration d'aluminium et du plomb (mg/l) en fonction des puits étudiés 


\section{Qualité globale des eaux souterraines de la région Sidi kacem- Sidi Slimane}

L’appréciation de la qualité des eaux souterraines s'appuie sur l'étude des paramètres de pollution, et l'interprétation des résultats sur la base d'une grille simplifiée (tab.1) tenant compte de trois paramètres; indicateurs de pollution physico-chimique et azotée qui sont la conductivité, le taux des chlorures et celui des nitrates.

La conductivité électrique et les concentrations en ions chlorures renseignent sur la qualité minéralogique des eaux alors que les nitrates sont les principaux indicateurs d'une pollution d'eau souterraine.

L'examen des différents paramètres cités au niveau des stations analysées a permis de classer les échantillons suivant leur état de qualité (tab.2).

Tableau.1: Grille simplifiée pour l'évaluation de la qualité globale des eaux souterraines

(Ministère chargé de l'aménagement du territoire, de l'eau et de l'environnement, 2001)

\begin{tabular}{|c|c|c|c|}
\hline \multirow{2}{*}{} & \multicolumn{3}{|c|}{ Paramètres } \\
\cline { 2 - 4 } & $\begin{array}{c}\text { Conductivité électrique } \\
(\mu \mathrm{s} / \mathrm{cm})\end{array}$ & $\begin{array}{c}\text { Chlorures } \\
(\mathrm{mg} / \mathrm{l})\end{array}$ & $\begin{array}{c}\text { Nitrates } \\
(\mathrm{mg} / \mathrm{l})\end{array}$ \\
\hline Excellente & $<400$ & $<200$ & $<5$ \\
\hline Bonne & $400-1300$ & $200-300$ & $5-25$ \\
\hline Moyenne & $300-2700$ & $300-750$ & $25-50$ \\
\hline Mauvaise & $2700-3000$ & $750-1000$ & $50-100$ \\
\hline Très mauvaise & $>3000$ & $>1000$ & $>100$ \\
\hline
\end{tabular}

Tabeau 2: Qualité globale des eaux souterraines étudiées

\begin{tabular}{|c|c|c|c|c|}
\hline Échantillon & NO3 $^{-}$ & $\mathbf{C l}^{-}(\mathbf{m g} / \mathbf{l})$ & Conductivité $(\boldsymbol{\mu s} / \mathbf{c m})$ & Qualité \\
\hline E1 & 0,669 & 541 & 3000 & Très Mauvaise \\
\hline E2 & 30,5 & 149 & 1470 & Moyenne \\
\hline E3 & 49,084 & 376 & 2560 & Moyenne \\
\hline E4 & 5,414 & 350 & 1961 & Moyenne \\
\hline E5 & 44,814 & 642 & 3680 & Très Mauvaise \\
\hline E6 & 6,200 & 212 & 1440 & Moyenne \\
\hline E7 & 3,569 & 802 & 3450 & Très Mauvaise \\
\hline E8 & 29,870 & 518 & 2850 & Mauvaise \\
\hline E9 & 93,522 & 2734 & 9890 & Très Mauvaise \\
\hline E10 & 5,670 & 1422 & 4900 & Très Mauvaise \\
\hline E11 & 0,031 & 110 & 925 & Bonne \\
\hline E12 & 5,690 & 631 & 2780 & Mauvaise \\
\hline E13 & 2,700 & 105 & 770 & Bonne \\
\hline E14 & 160,000 & 750 & 4050 & Très Mauvaise \\
\hline E15 & 25,000 & 153 & 1090 & Moyenne \\
\hline E16 & 6,130 & 202 & 1045 & Bonne \\
\hline E17 & 5,17 & 321 & 2800 & Mauvaise \\
\hline
\end{tabular}




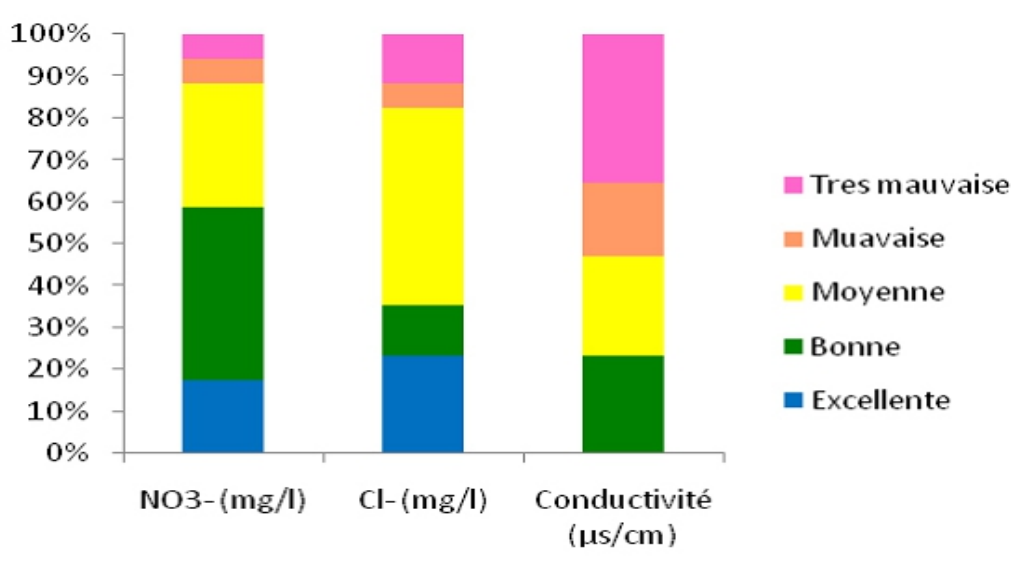

Figure 9: Histogramme de l'état de la qualité des eaux souterraines étudiées

Les eaux des échantillons E11, E13, E16 (tab.1) présentent globalement une bonne qualité pour les paramètres suivants :

- $\quad$ la minéralisation, avec des valeurs maximales enregistrées de 1090 $\mu \mathrm{S} / \mathrm{cm}$ (qualité bonne) ;

- $\quad$ Les chlorures sont très faibles (qualité excellente);

- $\quad$ Les nitrates varient entre un minimum de $0,031 \mathrm{mg} / \mathrm{l}$ et un maximum de 6,13 mg/l (qualité bonne).

L'analyse des échantillons E2, E15, E5 et E3 montre que la qualité globale de l'eau est moyenne en raison des teneurs en nitrates qui oscillaient entre $25 \mathrm{mg} / \mathrm{l}$ et $50 \mathrm{mg} / \mathrm{l}$ (tab.1).

Les autres points analysés sont de qualité mauvaise à très mauvaise, liée aux sels dissous et aux fortes teneurs en nitrates qui dépassent les normes marocaines.

La qualité globale des eaux souterraines de la région Sidi Kacem-Sidi Slimane peut être qualifiée d'assez bonne pour près de la moitié des stations étudiées. Malheureusement, un peu plus de la moitié (53\%) de ces eaux sont de mauvaise qualité voire très mauvaise, au vu de leur forte minéralisation et leur forte teneur en nitrates (fig. 10). 


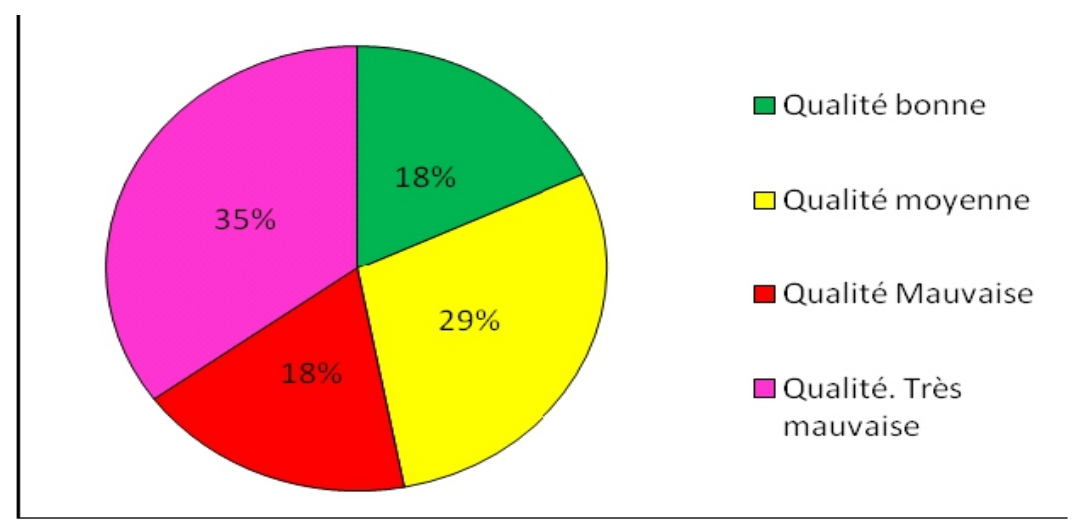

Figure10 : Récapitulatif de la qualité globale des puits étudiés

\section{Conclusion}

Les résultats obtenus dans cette étude ont permis d'évaluer la qualité physico-chimique et métallique des eaux souterraines de la région de Sidi Kacem-Sidi Slimane.

Les analyses physico-chimiques effectuées dans ce travail, ont montré que le $\mathrm{pH}$, l'oxygène dissout, le potassium, les orthophosphates et l'ion Ammonium peuvent être considérés admissibles et ne présentent aucune incidence sur la qualité des eaux. Ces résultats sont en accord avec ceux obtenus sur les eaux de la nappe phréatique de M’nasra (Zouhri, 2000; Zouhri et Carlier, 2002), du Gharb (Belksiri) (Akhiar, 2009) et de Fès (Elhammoumi et Belghyti, 2012).

Les métaux lourds dosés ont montré une contamination avec des valeurs assez importantes dans le puits E2 qui présente une concentration inquiétante en plomb $(0,133 \mathrm{mg} / \mathrm{l})$ et au niveau des puits E16 et E17 qui présentent une contamination en aluminium $(0,334$ et $0,452 \mathrm{mg} / \mathrm{l})$. Ceci serait causé par l'utilisation excessive des engrais et fertilisants dans le périmètre irrigué du Gharb.

L'analyse de la qualité globale des eaux souterraines de la région Sidi Kacem- Sidi Slimane montre que:

- $\quad 47 \%$ des points échantillonnés ont une minéralisation des eaux faible à moyenne et sont de qualité globalement moyenne à bonne alors que 53\% des points d'eau ont une minéralisation importante avec des valeurs de conductivité atteignant $9890 \mu \mathrm{s} / \mathrm{cm}$ ce qui leur confère une mauvaise à très mauvaise qualité ;

- $\quad$ Les teneurs en chlorures varient entre un minimum de 110 au niveau du puits E11 et un maximum de $2734 \mathrm{mg} / \mathrm{l}$ au niveau du puits E9. Certaines valeurs élevées (E9 et E10 montrant 2734 et $1422 \mathrm{mg} / \mathrm{l}$ respectivement) pourraient être mises en relation avec la composition naturelle du sol ; 
- $\quad 88 \%$ des puits ont des teneurs en nitrates inférieures au seuil fixé par les normes marocaines et $12 \%$ ont des teneurs supérieures et importantes, signe d'une incontestable pollution.

Dans l'ensemble, cette étude a permis de réaffirmer la pollution des eaux souterraines de la nappe du Gharb du fait de l'intensification agricole que connaît la région de Sidi-Kacem-Sidi Slimane. Face à cette situation alarmante qui s’inscrit malheureusement dans le temps, il est important de suivre la qualité de l'eau par des outils adaptés afin de corriger et préserver la nappe du Gharb qui reste une source importante pour l'alimentation en eau potable de cette région.

\section{References:}

Akhiar S. (2009) : Caractérisation des eaux souterraines de la ville de Mechraa Belksiri. Mém Master Sc. Environnement. Fac Sc. Kénitra.

Ben Abbou Mohamed, El Haji Mounia, Zemzami Mahmoud, Bougarne Loubna, and Fadil Fatima (2014) : Dégradation de la qualité des eaux de la nappe alluviale de l'Oued Larbaa par les déchets de la ville de Taza (Maroc) International Journal of Innovation and Scientific Research, Vol. 10 No. 2 Oct. 2014, pp. 282-294.

Bouchouata Ouafae, Brahim Jaafar, Moussa Bounakhla, Anass Doukkali, Benaïssa Attarassi (2011) : Etude de la contamination par les métaux lourds des eaux d'irrigation et les cultures maraîchères dans la zone M'nasra (Gharb, Maroc).ScienceLib Editions Mersenne : Volume 3, N ${ }^{\circ} 111006$.

Daifi H., Alemad A., Kherrati I., Elkharrim K., Khadmaoui A., Dlimi L., Lhoussin F., Belghyti D. (2014) : Evaluation de la qualité physico-chimique et bactériologique de la Nappe Maâmora (Kenitra-Maroc). Science Lib Editions Mersenne: Volume 6, N¹40902.

Daniane M, Chakor B. and Bensouda K., (1994) : Office régional de mise en valeur agricole du Gharb (ORMVAG)": Une journée débat sur : Les problèmes de l'environnement dans la région du Gharb (ORMVAG MAROC). Novembre (1994).

Direction Générale de l'Hydraulique (DGH) (1994) : Le point sur la sécheresse au Maroc.

El Blidi S., Fekhaoui M. (2003) : Hydrologie et dynamique marégraphique de l'estuaire du Sebou (Gharb, Maroc). Bulletin de l'Institut Scientifique, Rabat, 25 : 57-65.

Elbouqdaoui K., Aachib M., Blaghen M. et Kholtei S. (2009) : Modélisation de la pollution par les nitrates de la nappe de Berrechid, au Maroc, Afrique Sciences, 5(1), pp. 99-113, 2009.

El Hammoumi T.et Belghyti D. (2012) : Caractérisation physicochimique des eaux potables Produit Par la station de traitement de Mkansa (Maroc, 2012). ScienceLib Editions Mersenne : Volume 4, $\mathrm{N}^{\circ} 121201$. 
Ezzaouaq M. (1991) : Caractérisation hydrodynamique, physico-chimique et bactériologique des eaux superficielles de l'estuaire du Bouregreg (Maroc) soumis aux rejets des villes de Rabat-Salé. Thèse D.E.S. fac. Sci. Rabat, 140p.

Haddad H. et Ghoualem H, (2014) : Caractérisation physico-chimique des eaux du bassin hydrographique côtier algérois Larhyss Journal, ISSN 11123680, n¹8, Juin 2014, pp. 155-167.

HCEFLCD (Haut Commissariat aux Eaux et Forêt et la Lutte Contre la Désertification) (2007) : Etude diagnostique de la zone humide AL MassiraFaija, cercle d'EL Brouj et Cercle de Settat (Maroc), 242p.

Himmi N, Fekhaoui M., Foutlane A., Bourchic H., El Maroufy M., Benazzout T., et Hasnaoui M. (2003) : Relazione plankton-parametri fisici chimici in un bacino dimaturazione (laguna mista Beni Slimane - Morocco. Rivesta di idrobiologia. Universitadegli studi di Perugia, departemento di biologia animale ed ecologia laboratoire di idrobiologia “G.B. Grassi”, 110_ 111 .

Kholtei S. (2002) : Caractérisation physico-chimique des eaux usées de la ville de Settat et de Berrechid et évaluation de leur impact sur la qualité des eaux souterraines. Thèse de Doctorat d’État, Université Hassan 1er, Settat, Maroc, 162 p.

Kholtei S., A. Bouzidi, M. Bonin, M. Fekhaoui, R. Anane, K. Sbai et E. Creppy E (2003) : Contamination des eaux souterraines de la plaine de Berrechid dans la région de la Chaouia au Maroc par les métaux lourds dans les eaux usées : effets de la pluviométrie. Vecteur Environ., 36, 68-80.

Ministère chargé de l'aménagement du territoire, de l'eau et de l'environnement (2001) : Etat de la Qualité des Ressources en Eau au Maroc 2000/2001.

Moullard L. et Hazan R. (1960): Nappe phréatique de la plaine de Berrechid. Ass. Gen. Helsinki, Finlande, 25 juillet au 6 août, 52, 105-142.

N'diaye Abdoulaye Demba, Khadijettou Mint Mohamed Salema, Mohamed Brahim El Korya, Mohamed Ould Sid'Ahmed Ould Kankou et Michel Baudu (2014) : Contribution à l'étude de l'évolution Spatio-temporelle de la qualité physicochimique de l'Eau de la Rive droite du fleuve Sénégal. J. Mater. Environ. Sci. 5 (1) ISSN : 2028-2508 CODEN: JMESCN p. 325

O.M.S. (1986) : Organisation Mondiale de la Santé « Charte d’Ottawa pour la promotion de la santé», Copenhagen : Bureau régional de l'Europe, (1986).

OMS (2013) : Directives OMS pour l'utilisation sans risque des eaux usées, des excreta et des eaux ménagères, 2013. Volume 2, chapitre 8. p 125.

Pesson P., (1976). Pollution des eaux continentales, incidences sur les biocénoses aquatiques, gauthier villars, ISBN: 2-04-008704-4, p 97-101. 
Polan Patrick et Monique Henry (1990) : qualité de l'eau souterraine dans la MRC de Coaticook, Régie régionale de la santé et des services sociaux de l'Estrie direction de la santé publique et de l'évaluation.

Saaidi E. (1988) : Géologie du quaternaire marocain 439 p.

Saadi Z., A. Maaslouhi, M. Zeraouli et J. P Gaudet (1999) : Analyse et modélisation des variations saisonnières des concentrations en nitrates dans les eaux souterraines de la nappe Mnasra, Maroc, C. R. Acad. Sci., Sér. 2, Sci. Terre Planètes, 329 (8) (1999) 579-586.

Zouhri L. (2000) : Structure et modélisation hydrodynamique de l'aquifère de la Maâmora (Maroc), Thèse, Univ, Lille I, 218p.

Zouhri L. et Carlier E., (2002) : Caractérisation hydrochimique d'une nappe côtière, Maroc. Journal of Environmental Hydrology, Vol. 9, Paper 4, 1-7. 